\title{
ON THE OSCILLATIONS AND LEBESGUE CLASSES OF A FUNCTION AND ITS POTENTIALS ${ }^{1}$
}

\author{
BY R. K. JUBERG
}

Communicated by A. Zygmund, September 7, 1965

Suppose $f \in L^{r}(R), r \geqq 1, R$ a cube in $E^{n}$. Then one knows from Sobolev's theorems [5] that the potential

$$
P \rightarrow \int_{R} f(Q)|P-Q|^{-a n} d Q, \quad 0<\alpha<1,
$$

is in $L^{\sigma}(R), \sigma^{-1}>\alpha-1+r^{-1}$, where $|P-Q|$ denotes the Euclidean distance between $P, Q \in E^{n}$.

In this note we demonstrate a certain converse proposition. For a non-negative function $f \in L^{r}(R), r \geqq 1$, we assume the potential (0.1) to be in $L^{s}(R), 0 \leqq s^{-1}<\alpha-1+r^{-1}(s$ a positive real number or $\infty)$, and in addition make an assumption on the "oscillations" of $f$ (cf. $\S 1$ ). Then we can conclude that $f$ is summable to powers exceeding $r$.

We express the so-called "oscillatory" conditions and present the main theorem, Theorem A, in the next section. The proof of the theorem is direct and simple. In $\$ 2$ we state a parallel theorem, Theorem $\mathrm{B}$, wherein the assumption on the potential is replaced by the hypothesis that the function is in some "Morrey class" (cf. Morrey [3]; or also Campanato [1]). Theorem B is described perhaps more accurately as a corollary to the proof of Theorem A. In the last section, $\S 3$, we show how these results can be indirectly deduced. Therein we use a lemma from a paper by Semenov [4] which relates "Marcinkiewicz classes" (cf. e.g., Zygmund [6]) with "Lorentz" spaces. The conclusion follows then from the inclusion relations between Lorentz spaces and Lebesgue spaces (cf. Lorentz [2]).

1. The principal result. Let $f$ be a non-negative function summable over $R$, a cube in $E^{n}$. For $S$ any measurable set in $E^{n}$ we indicate its (Lebesgue) measure by meas $S$. Set

$$
E(x)=\{P: P \in R, f(P)>x\} .
$$

Condition I. For some $a>0,0 \leqq \lambda \leqq 1$ ( $a$ may depend on $\lambda$ )

1 This work was supported in part by the Office of Naval Research under Contract No. Nonr-710(54). 


$$
x(\text { meas } E(x))^{1+\lambda} \leqq \sup \int_{c} f(Q) d Q, \quad x>a,
$$

where the supremum is taken over all parallel subcubes $C \subset R$ with volume meas $E(x)$. Denote by $\bar{\lambda}$ the infimum of the set of numbers $\lambda$ for which (1.2) holds.

If one considers the inequality (1.2) for some fixed $x$, then it can be interpreted as a condition on the dispersion of the set of points where the function assumes large values (exceeding $x$ ). It is for this reason that we refer to the foregoing as a condition on the oscillations of a function (and, similarly, for the alternate conditions presented later in this section).

REMARK. One property of the quantity $\bar{\lambda}$ is that its reciprocal measures what one might describe as the upper bound (with respect to the exponent) of the Lebesgue classes of $f$. That is, $f$ is at best in $L^{1 / \pi}$. This observation, however, is seemingly not very interesting. For consider the situation on the line: $n=1, R$ and $C$ intervals. It is clear that for a monotone function $\bar{\lambda}=0$. Whereas there are monotone functions in $L^{p}$ and not in $L^{p+\bullet}$ for any $p$ and $\epsilon>0$.

Theorem A. ${ }^{2}$ Suppose $f \in L^{r}(R), r \geqq 1$, and the potential (0.1) is in $L^{s}(R)$ where $0 \leqq s^{-1}<\alpha-1+r^{-1}$. If $\bar{\lambda}<\alpha-1+r^{-1}-s^{-1}$, then $f \in L^{p}(R)$ for $p<\left(1-\alpha+s^{-1}+\bar{\lambda}\right)^{-1}$.

Proof. It follows simply using Hölder's inequality that

$$
\int_{C} \int_{R} f(Q)|P-Q|^{-\alpha n} d Q d P \leqq(\text { Constant) (meas } C)^{1-s^{-1}} .
$$

Now the left side in (1) dominates the quantity

$$
(\operatorname{dia} C)^{-\alpha n}(\text { meas } C) \int_{c} f(Q) d Q \text {. }
$$

From (1), (2) and the fact that (dia $C)^{n}=n^{n / 2}($ meas $C$ ) we find that

$$
\int_{C} f(Q) d Q \leqq(\text { Constant })(\text { meas } C)^{\alpha-s^{-1}} \text {. }
$$

Set $\lambda=\bar{\lambda}+\epsilon$ where $\epsilon>0$ is any number satisfying the inequality $\bar{\lambda}+\epsilon<\alpha-1+r^{-1}-s^{-1}$. Then on combining (1.2) and (1.3), for sufficiently large $x$, it follows that

${ }^{2}$ This theorem was presented in preliminary form at the 69th Summer Meeting, American Mathematical Society, Amherst, Massachusetts, August 25-28, 1964. Notices Amer. Math. Soc. 11 (1964), 574. 


$$
x(\text { meas } E(x))^{1+\lambda} \leqq(\text { Constant) (meas } E(x))^{\alpha-s^{-1}} ;
$$

or

$$
\text { meas } E(x) \leqq(\text { Constant })\left(\frac{1}{x}\right)^{1 /\left(1+\lambda-\alpha+e^{-1}\right)} .
$$

The desired conclusion results from (3), the boundedness of meas $E(x)$, and the fact that

$$
\int_{R}[f(Q)]^{p} d Q=p \int_{0}^{\infty}(\text { meas } E(x)) x^{p-1} d x .
$$

We shall present now two other conditions that can be used instead of Condition I. The three conditions are ordered according to increasing relative strengths.

Condition II. Let $f^{*}=f^{*}(t), 0<t<$ meas $R$, be a decreasing function equi-measurable with $f$. Set

$$
\mu=\limsup _{z \rightarrow 0} \frac{\log \left(\sup \int_{c} f(P) d P / \int_{0}^{z} f^{*}(t) d t\right)}{\log z}
$$

where the supremum in the numerator is to be taken over all parallel subcubes $C \subset R$ with volume $z$.

Condition III. Suppose meas $E(x)>0, x>0$. Set

$$
1+\nu=\limsup _{x \rightarrow \infty} \frac{\log [\sup \text { meas }(E(x) \cap C)]}{\log (\text { meas } E(x))}
$$

where sup meas $(E(x) \cap C)$ is taken over all parallel subcubes $C \subset R$ with volume meas $E(x)$.

REMARK. The theorem then holds with $\mu$ or $\nu$ in place of $\bar{\lambda}$.

We observe further that a more local type theory could be developed based on local conditions similar to the above. For example, consider Condition II: For $P$ fixed in $R$ formulate (1.4) for a cube with center $P$ and contained in $R$. Then shrink the cube down to $P$.

2. A parallel theorem. Let $S$ be a bounded open set in $E^{n}$ of diameter $\rho_{0}$. Denote by $B(P, \rho)$ the ball with center $P$ and radius $\rho$. Let $q$ and $\delta$ be real numbers where $q \geqq 1$ and $0 \leqq \delta \leqq n$. A function $f$ is said to be in the Morrey class $L^{(q, 8)}(S)$ if there exists a constant $K$ such that

$$
\int_{B(P, D) \cap s}|f(Q)| a d Q \leqq K \rho^{8}
$$


for all $P \in S$ and $0 \leqq \rho \leqq \rho_{0}$.

We apply this definition in a slightly modified form. Here $S$ is $R$, a cube in $E^{n}$. We take cubes $C=C(P, \rho)$ of diameter $\rho$ centered at points $P$ of $R$ instead of balls. Then we replace relation (2.1) by the equivalent relation

$$
\left(\int_{C \cap R}|f(Q)|^{q} d Q\right)^{1 / q} \leqq K(\operatorname{meas} C)^{\beta}
$$

where $0 \leqq \beta \leqq q^{-1}$. We denote the corresponding class now by $L^{(q, \beta)}(R)$.

TheOREM B. Let $f$ be a non-negative function in $L^{(q, \beta)}(R)$. If $\bar{\lambda}<\beta$ then $f \in L^{p}(R)$ where $p<\left(q^{-1}-\beta+\bar{\lambda}\right)^{-1}$.

The proof parallels that of Theorem A. Instead of relation (1.3) we deduce in this case using Hölder's inequality and (2.2) that

$$
\int_{C} f(Q) d Q \leqq(\text { Constant })(\text { meas } C)^{1-q^{-1}+\beta} \text {. }
$$

The proof is completed then just as in the proof of Theorem A.

3. An indirect proof. A function $f$ measurable on $R$ is said to be in the Lorentz space $M(\gamma), 0 \leqq \gamma \leqq 1$, provided that

$$
\|f\|_{M(\gamma)}=\sup _{0<\varepsilon<\text { meas } R} \frac{\int_{0}^{*} f^{*}(t) d t}{z^{\gamma}}<\infty
$$

where $f^{*}=f^{*}(t), 0<t<$ meas $R$, is a decreasing function equi-measurable with $|f|$.

We shall say that a function $f$ in $R$ is in the Marcinkiewicz class $\mathfrak{M}(\gamma), 0 \leqq \gamma \leqq 1$, if it satisfies the condition

$$
\sup _{0<x<\infty} x(\text { meas } E(x))^{1-\gamma}<\infty
$$

where meas $E(x)$ is the distribution function of $|f|$.

The following lemma which relates Lorentz spaces and Marcinkiewicz classes appears in [4].

LEMMA. The Marcinkiewicz class $\mathfrak{M}(\gamma)$ coincides with the space $M(\gamma)$. In addition

$$
\sup _{x} x(\text { meas } E(x))^{1-\gamma} \leqq\|f\|_{\mu(\gamma)} \leqq \gamma^{-1} \sup _{x} x(\text { meas } E(x))^{1-\gamma} .
$$


Now consider again the proof of Theorem A. We deduce from (3.2), using relation (3) in the proof, that $f \in \mathfrak{M}\left(\alpha-\lambda-s^{-1}\right)$. Then on applying the Lemma it follows that $f \in M\left(\alpha-\lambda-s^{-1}\right)$. The desired conclusion is derived finally from the inclusion relation $M(\gamma) \subset L^{\left(1-\gamma^{\prime}\right)^{-1}}$, $\gamma^{\prime}<\gamma$.

\section{REFERENCES}

1. S. Campanato, Proprietd di inclusione per spazi di Morrey, Ricerche Mat. 12 (1963), 67-86.

2. G. G. Lorentz, Some nerw functional spaces, Ann. of Math. 51 (1950), 37-55.

3. C. B. Morrey, Multiple integral problems in the calculus of variations and related topics, Univ. of California Publ. Math. (N.S.) 1 (1943), 1-130.

4. E. M. Semenov, $A$ scale of spaces with an interpolation property, Dokl. Akad. Nauk SSSR 148 (1963), 1038-1041 = Soviet Math. Dokl. 4 (1963), 235-239.

5. S. L. Sobolev, On a theorem of functional analysis, Mat. Sb. 4 (1938), 471-497; English transl., Amer. Math. Soc. Transl. (2) 34 (1963), 39-68.

6. A. Zygmund, Theorem of Marcinkiewicz concerning interpolation, J. Math. Pures Appl. 35 (1956), 223-248.

UNIVERSITY OF MINNESOTA 